prevention of hepatitis B virus infections involve hyperimmune globulin given following exposure and, more recently, pre- and post-exposure vaccination. Hepatitis B virus vaccine is a highly purified protein vaccine from the serum of chronic hepatitis B virus carriers. It is highly immunogenic and efficacious in preventing hepatitis B virus infections when given in three doses over six months. However, antibody production may be inadequate in immunocompromised patients, such as those with chronic renal failure or those receiving chemotherapy. The side effects of the hepatitis B vaccine have been very rare and are of a mild nature. No specific recommendations for the immunization of anaesthetists have been made as this occupational group does not fall into the high risk group for priorization of the use of this expensive vaccine (which is still in short supply). Developments to be expected in the future include improvements in the diagnosis of infectious carriers, through direct viral DNA detection. New biotechnology will produce a less expensive and more abundant vaccination in the near future. Also, the laboratory diagnosis of Delta agent will improve our awareness of this defective virus, which appears associated with a worse prognosis and more frequent development of chronic liver disease in hepatitis B virus infection.

The next virus of potential nosocomial acquisition by anaesthetists is the herpes simplex virus (HSV). HSV is a DNA virus which can cause a latent and often asymptomatic infection in addition to primary infection of skin and mucous membranes. A recent study has shown a high rate (14 of 46 patients) of excretion of herpes simplex virus type 1 in tracheobronchial secretions in the adult respiratory distress syndrome. Also, the modified appearance of herpetic gingivostomatitis in immunocompromised patients may be erroneously mistaken for chemotherapy induced mucositis. Thus, exposure to HSV infected oral secretions occurs frequently in the practice of anaesthesia. The hazard to medical personnel is the introduction of the virus through a percutaneous route such as cuts or abrasions, with development of herpetic whitlow. These HSV infected lesions are often mistakenly diagnosed as bacterial infections. However, failure to respond to antibiotics, surgical incision without improvement and the possible recurrence of these lesions, clearly distinguishes an herpetic whitlow. In addition to local and frequently systemic symptoms, which may be of a severe nature, days are lost from work to avoid the spread of virus to others, until the lesions have crusted. We have noted four HSV nosocomial infections among medical residents performing anaesthesia-related functions in two Winnipeg teaching hospitals over the past 18 months. The risk of HSV infection can be reduced by wearing gloves during any procedure requiring contact with oral secretions. Antiviral treatment is available for severe HSV disease.

Acquired Immunodeficiency Syndrome (AIDS) has been considered a potential occupational threat to health care personnel, although no instances of disease among medical staff related to work activity, have been reported. Because blood and close physical contact have been implicated in the spread of AIDS, precautions similar to hepatitis B virus have been developed for the prevention of nosocomial AIDS. With the exciting discovery of a retrovirus as a cause of AIDS, new diagnostic tests will soon be available for screening and identifying high risk patients. Also, the fact the AIDS agent is a member of the retrovirus family should eliminate anxieties about the potential of hepatitis $B$ vaccine to transmit AIDS. These retroviruses would clearly be inactivated by the purification steps for hepatitis $B$ vaccine.

These are but a few of the infection hazards to which anaesthesia personnel are exposed during their practice. Liaison with hospital infection control personnel and public health officials is useful to recognize many of these viral hazards, and to provide prevention and control mechanisms for practicing anaesthetists.

\section{The impaired physician}

To the majority of physicians without past personal experience this topic may seem as new and unusual as Legionnaire's Disease. Very few medical schools deal in depth with the subject of drug and alcohol abuse; almost none couple such efforts with the concept of physician well-being. As a consequence, 
the very idea that doctors might fall prey to alcoholism or other chemical abuse generally seems alien. To further suggest that perhaps $10-14$ per cent of physicians in practice at some time in their careers will have problems with alcohol and/or other chemicals appears totally unacceptable. However, despite the subject's unpleasantness, and the difficulty in obtaining solid data, this estimate appears fairly accurate. One might reasonably well ask a number of questions concerning such a new/old, rare/prevalent problem, depending to some extent on whether one's personal response to the preceding suggestion is outright rejection or earnest concern (with perhaps a modicum of curiosity). This article is directed to those who have experienced the latter response.

First and foremost, where did this entire idea come from, and is it that new? Given that little mention of it has been, or is being made in most North American medical schools, the problem would appear to be absolutely new-born. The facts, however, belie this. Literature describing chemical abuse by physicians was well known in psychiatry by the 1960's. A substantially larger audience was exposed to the concept in 1973 when the Council on Mental Health of the American Medical Association published "The Sick Physician." This landmark article originated a nationwide movement in the United States of America complete with bi-annual conferences, concerned with the recognition and rehabilitation of the impaired physician. During the ensuing years since this important article, a substantial amount of literature has accumulated concerning various aspects of this problem. One of the most significant was that of Herrington $e t$ al. in which the unusual theory of a chemically-impaired doctor returning to practice, no matter the specialty, was proven sound. Other contributions especially relevant to anaesthesia, were made by Gravenstein, Ward and Spiegelman. Without attempting an exhaustive review of these many contributions, the following synopsis can be presented.

Alcoholism occurs at essentially the same rate amongst physicians as in a matched socioeconomic group (seven per cent). Non-alcohol chemical abuse occurs amongst physicians at a rate at least seven times greater than a matched socioeconomic group (two per cent - a figure put in stark perspective by the rapidly enlarging chemical abuse problem in the general U.S. population), anaesthesiologists appear to be represented at a rate 3-4 times greater than predicted by random physician distribution. In series reported from treatment programs, and rehabilitation of chemically impaired physicians, anaesthesiologists included, 60 80 per cent will return to successful practice.

From the preceding synopsis, a significant factor has been deleted, because its significance mandates more extensive comment. This missing factor is the disease concept of chemical dependency. Addiction generally brings to mind chronic daily and rapidly increasing use of chemicals associated with physical dependency. Withdrawal from this situation is most often perceived as immediate, violent and highly physical. This simple concept was thrown into disarray by the large number of U.S. servicemen who returned from Southeast Asia with significant drug use patterns, only to then abruptly cease drug use in the U.S. without - miraculously medical help. Furthermore, the generally described pattern of withdrawal hardly begins to encompass the profound psychological upset associated with cessation of cocaine abuse. These shortcomings, along with a number of older problems such as how to classify spree-drinkers, have led to a much different understanding of chemical dependency, emphasizing abuse rather than addiction. This disease of chemical abuse appears to be a very complex interaction of physical, social and psychological factors, modified by environment and genetics. The disease is chronic, almost invariably progressive, and commonly fatal.

One must coincidentally be aware, however, that a significant number of people, "expert" and otherwise, take major issue with this disease concept, arguing that a disease cannot be invoked as cause for voluntary ingestion of alcohol or narcotics. The pragmatic retort to this objection is that the disease concept allows for prediction of behaviour and suggestions for therapy - or, in essence, it works. Prior and current alternatives, no matter how sacrosanct or ancient, do not. Any physician who has been exposed to traditional, generally hopeless, approaches to a hospitalized chronic alcoholic can recognize this failure. Consequently, an optimistic and aggressive approach to the impaired physician must be based from the outset on a different 
approach to chemical dependency, that of a multifactorial disease, lest preconceived notions bring about avoidance and withdrawal.

What or who is an impaired physician? For identification purposes, one might wish for major abrupt aberrations in dress, appearance, language, hygiene and professional activity at the first sign of alcohol or chemical abuse. Unfortunately, until the very end stages of the disease, none of this is true. The impaired physician lives in a world held together by denial, so he or she is unaware and will not seek help. Furthermore, the hospital is the last environment in which impairment is manifest.

The disease first appears as gradual withdrawal from usual patterns of social, religious and leisure activities. Family life is degraded and embarrassing social episodes, such as arrests for driving while intoxicated, may occur. Personal health may become a source of ill-defined complaints. Legal problems, including impulsive poorly thought-out business arrangements, often further complicate matters. As the disease progresses further, hospital practice eventually becomes affected. Initially, this appears a subtle extension of ongoing problems. A previously involved, available, doctor becomes isolated and hard to locate; tardiness and absenteeism, usually well "excused," become common behaviour for someone who was previously never late or missing when needed.

Anaesthetic records become shoddy and drug requests may be unusual - provided there is a mechanism to review either. However, no matter how elaborate a control or review process available in any department, it is critical to realize that the very best one can hope for from any such system is to reemphasize the importance of the subject and/or possibly confirm a presumptive diagnosis of impairment. Despite a specialty infatuation with technical solutions, currently generally requiring computer involvement, primary reliance on any such checkout or restriction system for diagnosis, or worse still, elimination, of this disease, is destined to fail in a very self-serving fashion - generally, no one will be discovered to be impaired. This is because the polished manipulative skills of the abuser, in conjunction with the solo nature of anaesthesia practice in a busy OR environment, combine all too readily to defeat any practical system thus far suggested. In part, this may be because anaesthesi- ologists and OR nurses are rarely trained in police techniques. It is the author's firmly held opinion that drug accounting and control and anaesthetic record review, while undoubtedly needed and worthwhile, will never serve as more than adjuncts to awareness and observation for diagnosis.

Following individual recognition, usually due to changes in personal behaviour, what then? An instinctive usually terribly wrong response is to confront the impaired colleague one-on-one. Those same well-honed manipulative skills that allowed the chemically impaircd physician to defeat a drug accounting system will control such a person-toperson dialogue in a way that makes the confronting doctor feel he or she is the impaired one, for so falsely accusing a trusted friend who has a good reason for everything, explained so well that one can hardly doubt the veracity. After a bit of experience, one gradually comes to the realization that no one has a reason for everything; however, even with such experience, solo confrontation is ill-advised. Instead, a nonjudgmental, caring statement of facts, by several concerned colleagues to the impaired individual, should take place. The process is called intervention and, properly performed, is highly successful in getting impaired physicians into treatment.

The treatment, to be successful, must be tied to a physician with extensive experience caring for impaired health care professionals. To the unexperienced therapist, a chemically impaired physcian represents a barely tangible wraith of lies and half-truths, occasionally thought due to lack of chemicals rather than over-use. A more reasonable path is to search for a consultant well in advance of a specific need, such that a Saturday morning intervention requires a simple phone call, rather than an hour of frantic pleas for help to a dozen strangers.

After successful therapy, the recovering doctor must begin reentry, the long road back. Alcoholics Anonymous, Narcotics Anonymous and peer support, along with continued psychotherapy, are the mechanisms required to help reestablish self esteem and reinforce the basic honesty the recovering physician must obtain to return to work. The multiple patterns involved in this reentry process are beyond the scope of this paper.

At this juncture, one might reasonably ask tough, but legitimate, questions: what's all this got to do 
with me? Given that this problem may involve one physician in ten, why shouldn't the other nine simply identify the guilty and get rid of him or her? Or better still, ignore the whole subject and let others take care of it. To begin the response, the public at large has been bombarded with stories of various professionals, athletes, executives or doctors who are involved with drugs. To continue to maintain a semblance of public trust and confidence, the least accountable such profession is expected to take steps to attend to drug problems and support their own people through rehabilitation. Certainly medicine, above all other professions, must take a strong advocacy role for impaired physicians. Allowing others to attend to this task will, at the legislative level, unquestionably be painful.

At the local practice level, one runs afoul of the concept of vicarious liability, by which physicians can be held accountable for a colleague's misdeeds if they should have had reason to have known of impairment. In other words, "I didn't see a thing" is not a reasonable last-ditch defence.

Finally, since knowledge does not confer immunity, how can one be absolutely sure of resting comfortably amongst the disease-free, rather than being the unfortunate impaired individual? Given any insight whatsoever, each of us must treat an impaired colleague in the same fashion we would wish to be treated. When this potentially lethal disease is recognized, diagnosed and treated, those responsible will have participated in saving the life of a friend and fellow physician; to those who have experienced this, it represents one of the pinnacles of a medical career.

\section{References}

1 Modlin HC, Montes A. Narcotics addiction in physicians. Am J Psychiat 1964; 121: 358-65.

2 a'Brook MF, Hailstone JD, McLauchlan IEJ. Psychiatric illness in the medical profession. $\mathrm{Br}$ J Psychiat 1967; 113: 1013-23.

3 Putnam PL, Ellinwood EH Jr. Narcotic addiction among physicians: A ten-year follow-up. Am J Psychiat 1966; 122: 745-8.

4 Vaillant GE, Brighton JR, McArthur C. Physicians' use of mood-altering drugs. N Engl J Med 1970; 282: 365-70.

5 Little RB. Hazards of drug dependency among physicians. JAMA 1971; 218: 1533-5.
6 Vaillant GE, Sobowale NC, McArthur C. Some psychologic vulncrabilitics of physicians. N Engl J Med 1972; 287: 372-5.

7 AMA Council on Mental Health: The sick physician: Impairment by psychiatric disorders, including alcoholism and drug dependence. JAMA. 1973; 223: 684-7.

8 Rose KD, Rosow I. Physicians who kill themselves. Arch Gen Psychiat 1973; 29: 800-5.

9 Bissell $L$, Jones $R W$. The alcoholic physician: A survey. Am J Psychiat 1976; 133: 1142-6.

10 Johnson RP, Connelly JC. Addicted physicians: A closer look. JAMA 1981; 245: 253-7.

11 Chappel JN. Physician attitudes toward distressed colleagues. West J Med 1981; 134: 175-80.

$12 \mathrm{McCue} J D$. The effects of stress on physicians and their medical practice. N Engl J Med 1982; 36 : 458-63.

13 Herrington RE, Benzer DG, Jacobson et al. Treating substance-use disorders among physicians. JAMA 1982; 247: 2253-7.

14 Scheiber SC, Doyle BB. The Impaired Physician. New York, Plenum Medical Book Co., 1983.

15 Gualtieri AC, Cosentino JP, Becker JS. The California experience with a diversion program for impaired physicians. JAMA 1983; 250: 226-9

16 Gravenstein JS, Dory WP, Marks RD. Drug abuse by anesthesia personnel. Anesth Analg 1983; 62: 467-72.

17 Newman RG. Sounding Board. The need to redefine "addiction." N Eng J Med 1983; 308: 1096-8.

18 Ward CF, Ward GC, Saidman LJ. Drug abuse in anesthesia training programs. JAMA 1983; 250: 922-5.

19 Spiegelman WG, Saunders L, Mazze RI. Addiction and anaesthesiology. Anesthesiology 1984; 60: 335-41.

20 Henderson $H W$. Addicted doctors: Responding to their needs. Can Fam Physician 1983; 29: 1691-9.

21 Niven RG, Hurt RD, Morse RM et al. Alcoholism in physicians. Mayo Clin Proc 1984; 59: 12-16.

22 Morse RM, Martin MA, Swenson WM et al. Prognosis of physicians treated for alcoholism and drug dependence. JAMA 1984; 251: 743-6. 